\title{
Una company town dividida entre la frontera de México y Estados Unidos: Revalorización de los primeros trazados industriales de Mexicali y Calexico 1902
}

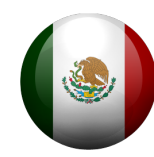

\section{Enrique Esteban Gómez Cavazos}

Arquitecto por la Universidad Autónoma de Nuevo León, en Monterrey, México. Master en investigación en Urbanismo. Estudiante de doctorado en Urbanismo por el Programa de Doctorado de Universitat Politécnica de Catalunya, en Barcelona, España. San Nicolás de los Garza [Nuevo León], México. <eecavazos@hotmail.com>

\section{Resumen}

Este artículo nos acerca al origen urbano de las ciudades de Mexicali y Calexico que comparten frontera entre México y Estados Unidos. La ciudad del lado mexicano, Mexicali, estuvo en sus inicios más conectada a Estados Unidos que a México. Se buscan los trazados originales de las compañías urbanizadoras y como la llegada del ferrocarril y los complejos sistemas de irrigación desde el Río Colorado dan inicio al crecimiento urbano del Valle Imperial en Estados Unidos y al Valle de Mexicali en México. Se destaca la importancia de las edificaciones pioneras del centro urbano y lo poco que queda de las construcciones industriales, defendiendo la hipótesis de que este territorio se ordena diferente al resto del país y que el patrimonio industrial actual puede ser relevante en la transformación de la ciudad en base a su identidad.

\section{Palavras-chave}

Patrimonio industrial. Identidad. Centro histórico.

\section{A company town divided between México and the United States border: Revaluation of the first industrial layouts of Mexicali and Calexico 1902}

\begin{abstract}
This article tries to approach the urban origin of the cities of Mexicali and Calexico located in the border they share between Mexico and the United States on the geographical beginning of the peninsula of Baja California. The city of Mexicali in the Mexican side was in its beginnings better connected to the United States instead of Mexico. The original urban grids that the companies develop arrive with the railroad and the complex irrigation system from the Colorado River, this started the urban growth: in the Imperial Valley in the United States and the Mexicali Valley in Mexico. We put special interest in the first industrial buildings in the city center defending the hypothesis that the industrial heritage can be important in the transformation of the city based on their identity.
\end{abstract}

\section{Keywords}

Industrial heritage. Identity. Historic center. 


\section{Introducción}

En la península de Baja California a finales del siglo XIX y principios del siglo XX se manifiesta un crecimiento urbano basado en un fuerte modelo de desarrollo económico. Se empieza a presentar un fenómeno urbanístico diferente al resto del país a través de concesiones territoriales de capitales extranjeros, en su mayoría estadounidenses, que modifican e influyen en la región con los trazados, arquitectura e industrias que implementan. Esto refuerza la economía de Estados Unidos y supone en México una lenta, pero ansiada ocupación urbana de la frontera norte.

Varias ciudades industriales surgen a partir del proyecto territorial del Porfiriato (1876-1910), que consiste en poblar, modernizar e industrializar los territorios más alejados del centro del país. De esta manera, las ciudades de las compañías, en este caso la aparición de la ciudad de Mexicali por inversiones de la Colorado River Land Company, la California Development Company y la Colorado Irrigation Company - compañías con fines agroindustriales - surgen de la visión de capitalistas extranjeros que crean la infraestructura necesaria para la industria; trazando centros de población conectados con el ferrocarril hacia Estados Unidos para la exportación de productos (como el algodón en el caso de Mexicali). Este tipo de ciudades se convierten, como muchas otras en esta región norte, en una característica urbana del régimen porfirista. El ferrocarril se vuelve fundamental y se convierte en vehículo de la modernidad arquitectónica, trayendo a estos territorios nuevas maneras de construir.

El objetivo principal de este texto es dar a conocer un caso de estudio como muestra de la industrialización por capital extranjero en la península de Baja California, argumentando una identidad diferente en el trazado urbano y en las edificaciones de esta parte del país con respecto a otras regiones. Se explica cómo se ordena el territorio y los comienzos del urbanismo en esta región de México buscando en su trazado original las piezas que puedan representar un potencial para mejorar su centro urbano, el cual hoy en día se encuentra en grave deterioro.

Este artículo forma parte de una investigación en curso titulada "Las ciudades de las compañías como modelo de urbanización en la península de Baja California", que analiza diversas poblaciones industriales de nueva fundación diseñadas por compañías extranjeras, la mayoría durante el Porfiriato. Se defiende la importancia de proteger el trazado original y las edificaciones que modernizaron esta región, cuestionando si las ciudades de las compañías constituyen un verdadero modelo de urbanización en el territorio, tomando esto como hipótesis general.

\section{Clasificación y breves apuntes tecnológicos}

Este corto apartado incluye los aspectos generales de la investigación en curso, abordando los procesos históricos de transformación urbana, identificando los valores patrimoniales a escala territorial, urbana y arquitectónica. Se muestra especial interés en compañías que instalan sus fábricas y que inmediatamente diseñan sus poblaciones. Se identifica en orden cronológico su origen y evolución.

La identificación y clasificación de compañías es posible gracias a la consulta de planos y archivos de las industrias, así como la revisión de documentos y publicaciones de autores como Antonio Padilla, David Piñera, William O. Hendricks, Dorothy Pierson Kerig y Cuauhtémoc Robles en el caso específico de Mexicali. Derivado del análisis y clasificación general del tipo de industrias establecidas en la península de Baja California.

Al día de hoy se han detectado por lo menos 10 casos donde compañías extranjeras han proyectado poblaciones en este territorio; este artículo estudia solo uno de ellos: el caso de Mexicali.

Mexicali cuenta actualmente con una traza original de edificios abandonados y degradados en su centro, con pocos ejemplos de edificaciones de balloon frame $e^{1}$. Lo contrario sucede en la población

\footnotetext{
${ }^{1}$ Sistema constructivo a base de estructuras prefabricadas de madera muy característico de Estados Unidos. Este tipo de construcciones se mostró particularmente adecuado para la colonización del Oeste americano, teniendo una influencia marcada en el norte de México.
} 
vecina de Calexico en Estados Unidos, donde se conservan varias viviendas antiguas de este sistema constructivo, de ahí la importancia de estudiar que tanto patrimonio existe del lado mexicano. Es necesario señalar que estos proyectos ayudan a construir el territorio peninsular, el cual cuenta con escasa población. La modernidad se desarrolla en ciudades de nueva fundación relacionadas principalmente con actividades económicas de productos para exportación.

\section{La gesta de las compañías para controlar el río Colorado}

A mediados del siglo XIX, el desarrollador de terrenos norteamericano, Oliver Wozencraft, cree que al introducir agua del río Colorado a la región desértica del delta -en el área que hoy comparte Mexicali y Calexico- éste podría convertirse en el centro agrícola más potente del sur de California. En un principio, la idea parecía imposible, sin embargo, después de su muerte en 1887, empresarios comenzaron a convencerse que esta idea podría ser una realidad.

El primer intento arranca con la Colarado River Irrigation Company pero colapsa en 1893 sin haber hecho un progreso notable. Un ingeniero de la compañía decide intentarlo de nuevo en 1896: Charles Rockwood, quien diseña el trazado de Mexicali y Calexico. Forma la California Development Company y contrata como jefe a George Chaffey -ingeniero canadiense quien ya había desarrollado con éxito dos colonias de riego en California- quien diseña en el Valle Imperial un canal de aproximadamente $80 \mathrm{~km}$ desde el río Colorado cerca de Yuma, al sur dentro de México y al este por el río Álamo, bordeando la frontera internacional.

El proyecto incluye una serie de compuertas a lo largo del canal. Las construcciones empiezan en 1900 y en un año el agua del río Colorado fluye a través del canal a las nuevas comunidades agrícolas en el Valle Imperial.

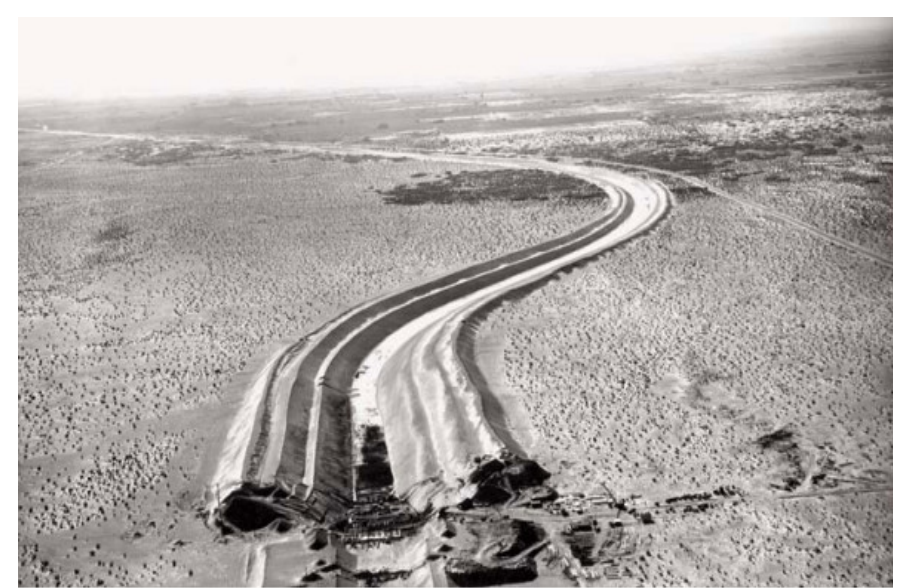

Figura 1. Imagen del canal todo americano en las primeras décadas del siglo XX, cruzando más de 80 kilómetros en el desierto para irrigar las primeras colonias agrícolas en la frontera entre México y Estados Unidos. Fuente: A century of service imperial irrigation district.

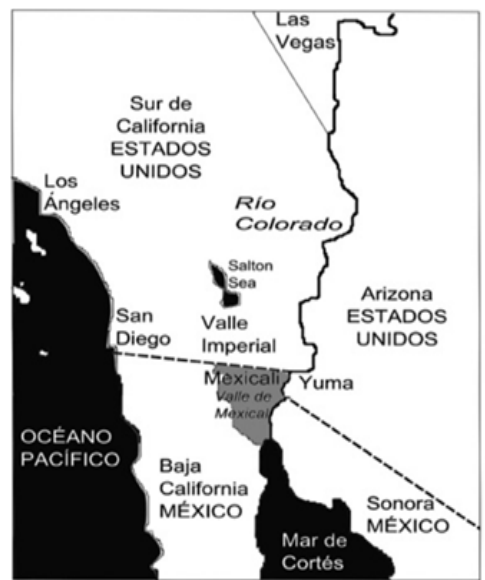

Figura 2. Localización del área de estudio, el territorio agrícola de Mexicali y Calexico. Fuente: Apostillas sobre la impronta simbólica del desierto territorio en la identidad cultural de Mexicali y su valle, de Hugo Méndez y Ernesto Israel Santillán.

La Colorado River Land Company, S.A. se establece como compañía mexicana en el año 1902, con el objetivo de utilizar las aguas desviadas del río Colorado para irrigar el Valle Imperial. Años después por medio de compras privadas, la compañía compra tierras en el ahora conocido valle de Mexicali asentándose en una región de poca población y de escasos servicios básicos. Años después adquiere 340.000 hectáreas de tierras desérticas, de las cuales 240.000 eran aptas para cultivo e irrigación (Kerig, 2001).

En 1904, es evidente que el canal no cuenta con la inclinacion suficiente, ya que la sedimentacion se acumula y el flujo de agua se desborda. Durante más de dos años, el río Colorado se derrama en el Valle Imperial, inundando en el lado norteamericano la mina de sal, las pequeñas poblaciones iniciales de El Centro, Calexico y la estación ferroviaria de Salton. En el lado mexicano, Mexicali 
también sufrió graves consecuencias ya que la mitad de su trazado se vio destruido, entre ellas la línea del ferrocarril, la cual ya se encontraba en funciones siendo propiedad del Ferrocarril del Pacífico Sur.

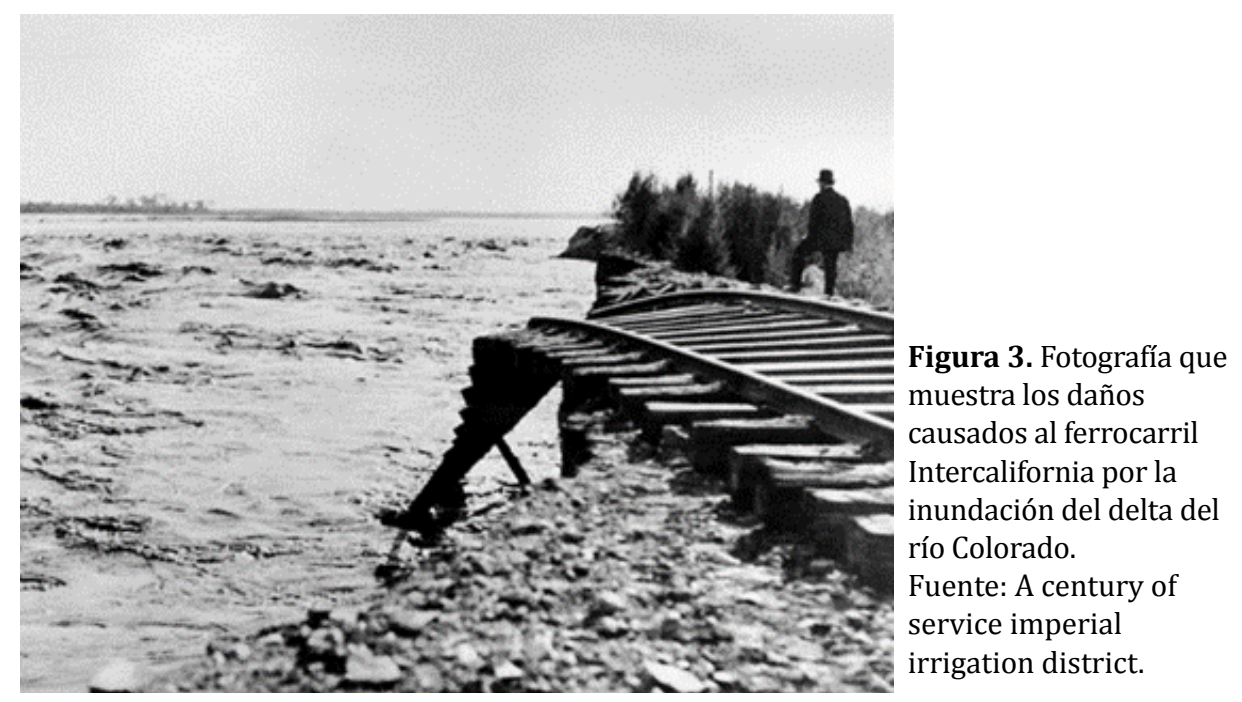

Los cambios bruscos de nivel en la zona crean cascadas en El río Álamo que acertadamente, después de la inundación, se le denomina río Nuevo. En la parte norteamericana, en Calexico, el Salton Sink, el cual era una laguna salada y seca se llena de agua con esta inundación, convirtiéndose ahora, en el lago más grande de California, un lago salado.

En la primera década del siglo XX se logra controlar al gran río Colorado, y se puede concluir que la visión de Wozencraft de irrigar el Valle Imperial se hizo realidad, esto a través de las compañías y el gobierno estadounidense, quien decide hacerse cargo de la gestión del río Colorado para evitar futuras inundaciones a través de un sistema de presas fortificadas.

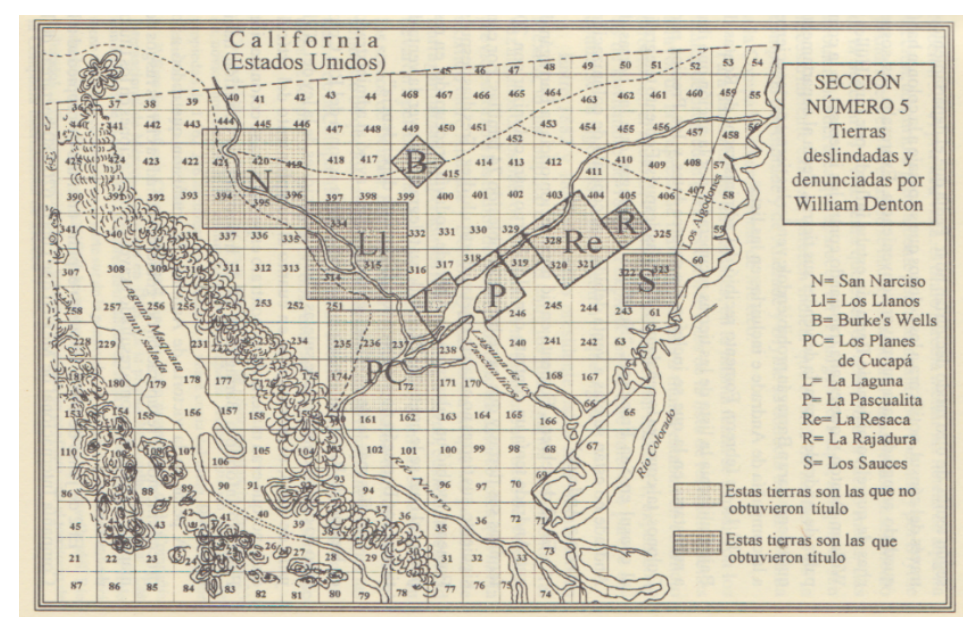

Figura 4. Plano que muestra las etapas de la venta de los terrenos propiedad de Guillermo Andrade en tiempos del Porfiriato a la Colorado River Land Company. Nótese que los terrenos abarcan todo lo que fue el valle de Mexicali en el delta mexicano del río Colorado. De ahí la importancia que tenía este territorio. Andrade ya había fundado la población de Lerdo en 1874, una pequeña colonia agrícola de más de 250 habitantes, (hoy abandonada). Richard E. Lingenfelter, en su libro, dice: "Aquí, en una mesa al este del río, estaba establecida Colonia Lerdo, un agradable pueblito con sus calles alineadas con eucaliptos". Al no tener Andrade suficiente capital y tecnología comparada con las compañías estadounidenses se vio en la necesidad de asociarse y vender para que ellas desarrollaran el delta del río Colorado. Fomentando las ideas y la estrategia de ocupar el territorio a través de concesiones a extranjeros. Fuente: Libro, "Guillermo Andrade y el desarrollo del delta mexicano del Rio Colorado 1874-1905” por William O. Hendricks. Editado por la UABC.

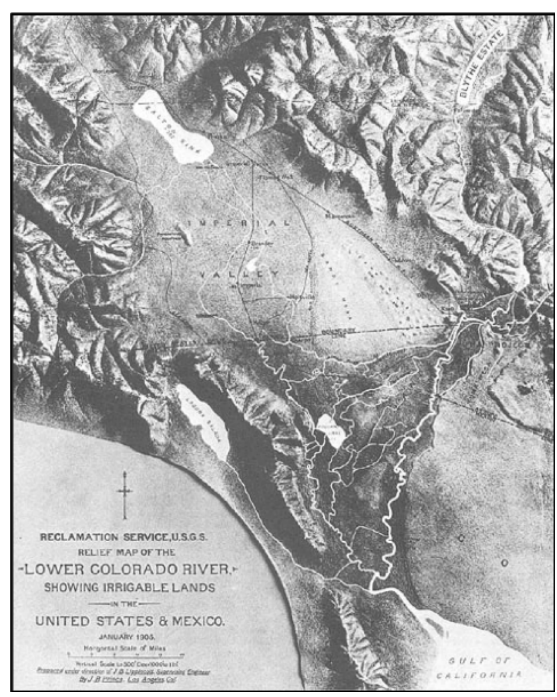

Figura 5. Mapa de 1905 del Imperial Valley en Estados Unidos y el Valle de Mexicali en México.

Fuente: U.S. Reclamation Service y U.S. Geological Survey. 
En 1928, se autorizan dos proyectos importantes para evitar futuras inundaciones: en primer lugar, se construye un canal dentro de la frontera internacional, el bien llamado, Canal Todo Americano. Éste, evitaría la construcción de otro canal en territorio mexicano. En segundo lugar, se establecieron ranchos de chinos, japoneses, hindúes y mexicanos, para consolidar el imperio agrícola en los dos lados de la frontera. Esto fue posible cuando se terminó de construir la presa Hoover, ya que controló el flujo del río Colorado haciendo posible el cultivo en terrenos que antes se inundaban, ubicados en la parte sur. Los primeros treinta años de la ciudad, corresponden al periodo en que se encontraba integrada a la economía del estado de California en Estados Unidos y aislada del resto de México. La Colorado River Land Company siguió teniendo mucho trabajo a inicios del siglo XX y como la inmigración proveniente del interior de México continuó, aumentó la presión de campesinos nacionales solicitando tierras para cultivo.

Así, en 1937, se suscita el llamado Asalto a las Tierras - el cual consistía en el reparto agrario por el entonces presidente Lázaro Cárdenas en donde el gobierno mexicano adquiere los terrenos de la compañía y los entrega a colonos mexicanos-. De esta forma, después de más de cuarenta años de posesión de casi todo el Valle de Mexicali, la Colorado River Land Company S. A. se retira de Baja California. Ésta compañía obtuvo beneficios de la explotación de la tierra y al salir dejó un legado a las generaciones siguientes: tierras apropiadas para el cultivo sin que el gobierno tuviera que invertir en el proceso (Kerig, 2001).

Es interesante ver como el desarrollo de la región fue diseñado por capitalistas estadounidenses en varias etapas. Primero: los trabajos de canalización de las aguas del río Colorado para irrigar el valle y hacer cultivable un desierto. Segundo: la planificación de los poblados y las diferentes maneras de urbanizar la frontera, ya que los planificadores trazaron en sus inicios una ciudad conjunta entre dos países, necesaria en ese momento para el funcionamiento urbano requerido para el desarrollo agroindustrial en la región fronteriza. Tercero: la conexión primera del ferrocarril, llevando estructuras de balloon frame para construir los primeros edificios y llevar gente para trabajar y cultivar la tierra, poblando así los dos lados de la frontera. Se considera llamativo el proyecto urbano inicial de Mexicali y Calexico ya que son pocos los casos que surgen al mismo tiempo en los dos lados de la frontera entre México y Estados Unidos. Es difícil imaginar que el Estado de Baja California ha sido gobernado por una ciudad que existe aproximadamente hace 113 años, y que la ciudad fue diseñada en la frontera por una compañía extranjera de desarrollo de terrenos llamada La Colorado River Land Company.

\section{Emigración y poblamento: una identidad diferente}

Lo que en la actualidad se conoce como el municipio de Mexicali, a principios del siglo XX era solo un desierto, "una porción de península que por varios siglos se consideró isla" (Aguirre, 1983), y que en un sentido físico realmente lo era, permaneciendo sin población ni desarrollo durante años, esto por su inaccesibilidad con el resto de México.

Los pioneros del asentamiento original de la población de Mexicali fueron de origen chino, llegaron aquí escapando de persecuciones xenófobas y los pocos que no eran perseguidos venían de paso sin intenciones de quedarse. A finales del siglo XIX se propició una fuerte inmigración de chinos hacia Estados Unidos con el fin de trabajar en la construcción de las vías férreas y en los canales de irrigación. Al terminar estas construcciones, el país del norte empezó a rechazarlos. Fue en 1904 cuando se emite una ley que prohíbe su entrada. Esto coincidió con el desarrollo agrícola de Mexicali, la cual requería de mano de obra y los habitantes de la región no eran suficientes para proporcionarla.

La comunidad asiática que llegó a trabajar en la región se asentó en el centro de la ciudad, fundando su propio barrio chino conocido como La Chinesca. Durante varios años, este barrio concentró la mayor parte de la población, esto debido a la cercanía con los campos de cultivo en el Valle Imperial. Siendo este barrió testigo de las mayores transacciones económicas de la región (Correa, 2014). 


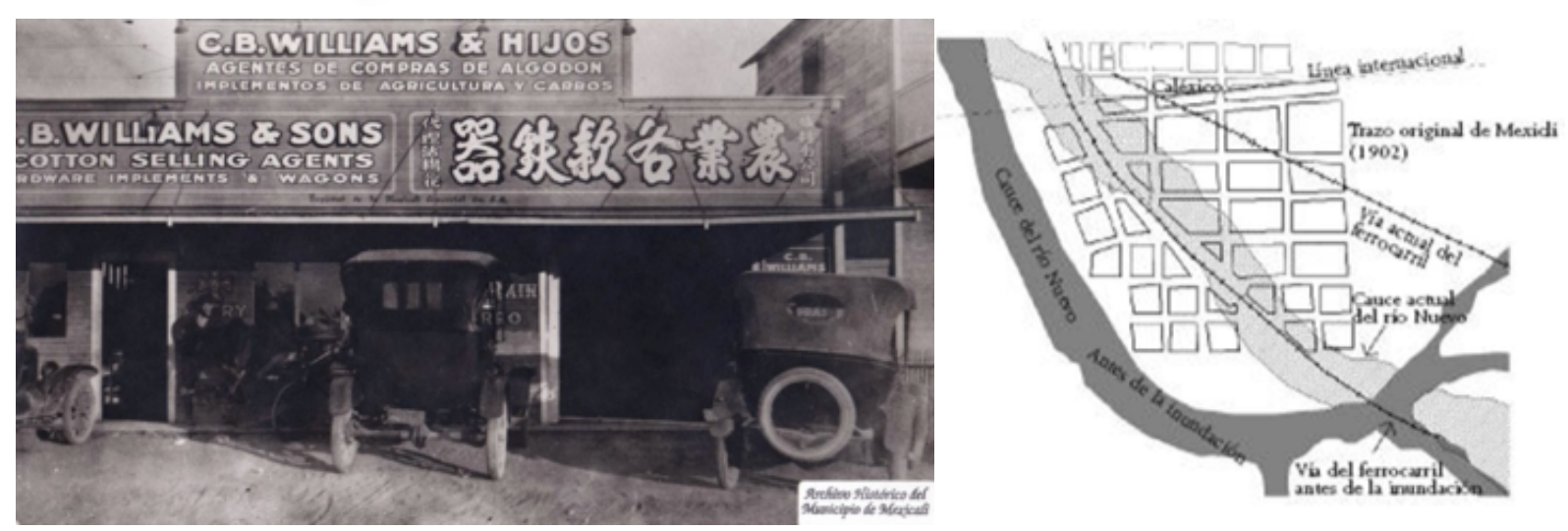

Figura 6. Fotografía de comercios chinos en Mexicali a principios del siglo XX, y plano obtenido del artículo "De la vulnerabilidad a la producción del riesgo en tres primeras décadas de la ciudad de Mexicali, 1903-1933". Dibujo Fuente: Barrientos (1982).

\section{Inicios de la Colonia Agrícola y de riego}

Los empresarios estadounidenses que tenían intenciones de asentarse en el Valle Imperial, particularmente en el límite fronterizo, sabían que el sistema de riego que corrió por primera vez en 1901 convertiría esas extensiones en un emporio agrícola, y que necesitaría de mucho trabajo para sostenerse. Así nació la ciudad de Mexicali, en simetría bilateral con Calexico su gemela gringa.

Como un primer intento de ordenamiento del espacio urbano en la frontera entre México y Estados Unidos, y con la idea de proyectar dos poblaciones simultáneas como núcleo de las inversiones estadounidenses en ambos valles, el ingeniero Charles Robinson Rockwood realiza el trazo de Mexicali conectado al de Calexico (Bernal, 2002).

El origen de los nombres de estas dos ciudades forman anagramas contrarios con las palabras: México y California. Calexico quiere decir "donde termina California y comienza México", mientras Mexicali significa lo contrario. La única separación física que existía entre estas dos ciudades era un montón de tierra. Conformaban una misma ciudad separada sólo por una frontera entre países. La diferencia entre estas dos ciudades radicaba en que, en Calexico vivían los jefes de las compañías, mientras que Mexicali era el lugar en donde se asentaban los trabajadores (Correa, 2014).

Figura 7. Esquema de ocupación del primer proyecto de las poblaciones en base al plano fundacional de 1902.

Fuente: Elaboración propia.

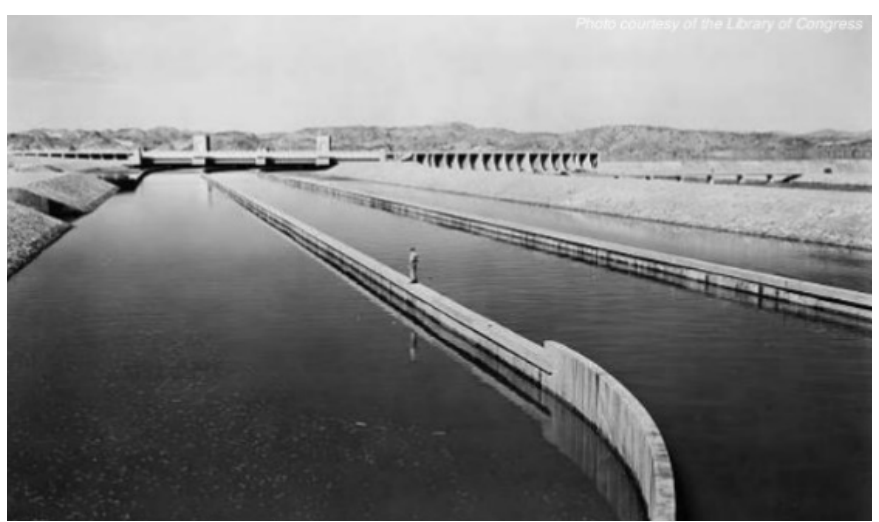

Figura 8. A inicios del siglo XX la construcción de este complejo sistema de irrigación en el desierto californiano fue el más grande y exitoso en el mundo. Fuente: From Imperial Dam to the Salton Sea: The story of Imperial Valley's water by Chris Austin. 


\section{Los primeros trazos urbanos}

El terreno donde nació el poblado mexicano no fue propiedad legítima de sus primeros colonos, sino de la Sociedad de Irrigación y Terrenos de la Baja California, una de las tantas compañías extranjeras que obtuvieron por parte del gobierno mexicano derechos de uso y explotación del suelo en Baja California (Robles, 2006).

En el trazado de Calexico en el lado estadounidense se aprecia una manzana tipo de $100 \mathrm{~m}$ x 115m aproximadamente, incluyendo su callejón en el lado más largo, unas manzanas más cuadradas que las rectangulares en el caso de Mexicali. En el lado mexicano, las manzanas eran aproximadamente de $180 \mathrm{~m}$ x 130m, también con su callejón en su lado más largo. Cabe mencionar que algunas de las calles coinciden a pesar de la frontera entre los dos países.

Esto indica que en sus inicios, las dos poblaciones conformaban un núcleo urbano continuo, donde las dos colonias agrícolas se complementaban. Actualmente las poblaciones están separadas por un muro de acero que las divide. Calexico se funda en 1899 como un campamento fijo de la Imperial Land Company, en 1902 se establece la oficina de correos y en 1908 se incorpora al estado de California, esta población sigue conservando su imagen de pueblo con su zona comercial paralela a la línea fronteriza y una tranquila sensación de amplitud. Pareciera que Calexico fuese una especie de gran fraccionamiento estadounidense con sus casas de madera bien ordenadas. Actualmente cuenta con 40.000 habitantes. En 1888, el gobierno federal mexicano otorga una gran parte del estado de Baja California, incluyendo Mexicali, a Guillermo Andrade (personaje importante en el desarrollo del Delta mexicano del Río Colorado), con el fin de colonizar la zona en la frontera de reciente creación con Estados Unidos. En 1900, la Compañía de Desarrollo de California con sede en EE.UU. recibió el permiso del gobierno de Porfirio Díaz para construir los canales de irrigación y atraer a los agricultores, naciendo así la población de Mexicali fundada en 1903. Hoy con casi 700.000 habitantes Mexicali rompió sus límites planeados y se consolida como la capital estatal más al norte de México.

El trazo más antiguo de Mexicali data de 1904 con la llegada del ferrocarril Intercalifornia y ubica el primer núcleo urbano en el ángulo sureste del cruce del río Nuevo con la línea internacional. El trazo de las manzanas rectangulares se separa del borde fronterizo mediante una franja de espacios vacíos. El esquema distributivo es sólo el registro catastral de 21 manzanas grandes que alinean las construcciones previas, y sugiere la compraventa de lotes hacia el sur y sureste en 12 manzanas más reducidas, aun siguiendo la misma orientación nortesur y oriente poniente (Mendez, 1993).

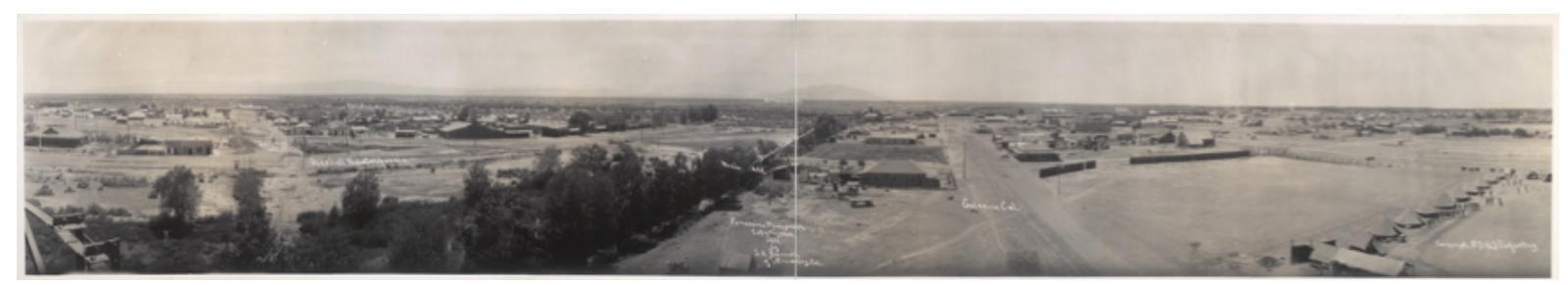

Figura 9. Fotografía de 1911 de las 2 poblaciones entre la frontera de México y Estados Unidos, se puede apreciar en el lado izquierdo Mexicali en Baja California México y en el lado derecho Calexico en California Estados Unidos. Fuente: Biblioteca del Congreso de Estados Unidos. 


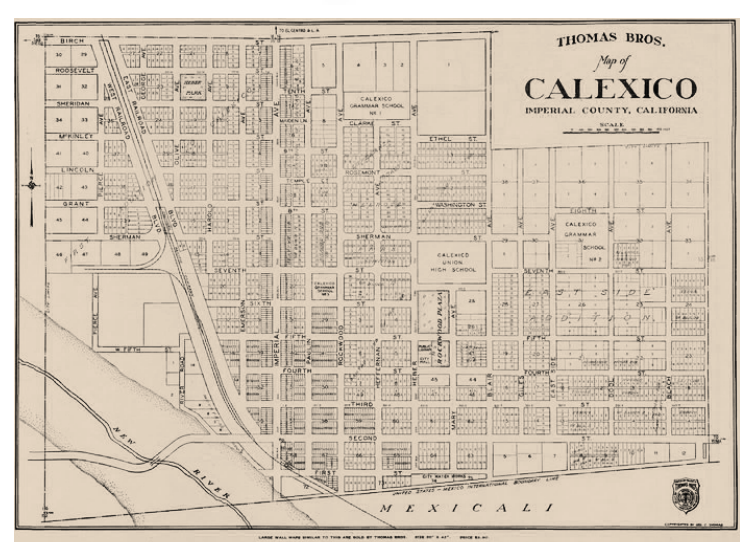

Figura 10. Plano de Calexico, Estados Unidos en 1920. Fuente: Barry Lawrence Ruderman Antique Maps Inc.

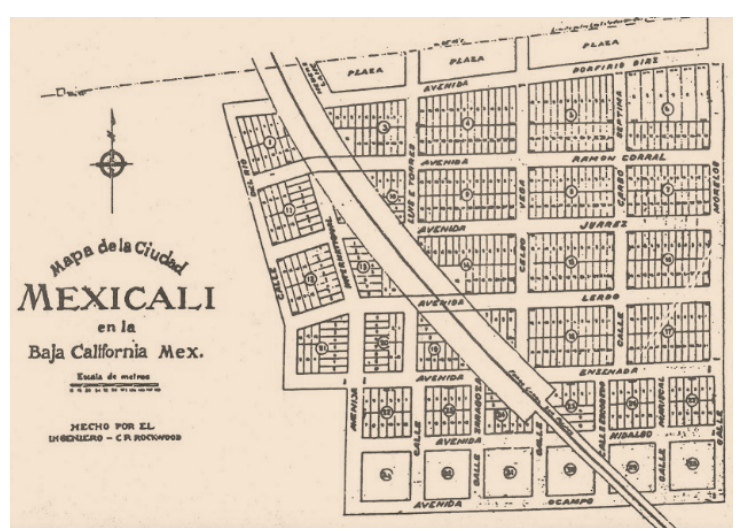

Figura 11. Plano de Mexicali en 1903. Fuente: Barry Lawrence Ruderman Antique Maps Inc.

Cuauhtémoc Robles en su libro La Arquitectura de Mexicali, hace referencia a lo dicho por el historiador y arquitecto Antonio Padilla Corona (1998), el cual expone:

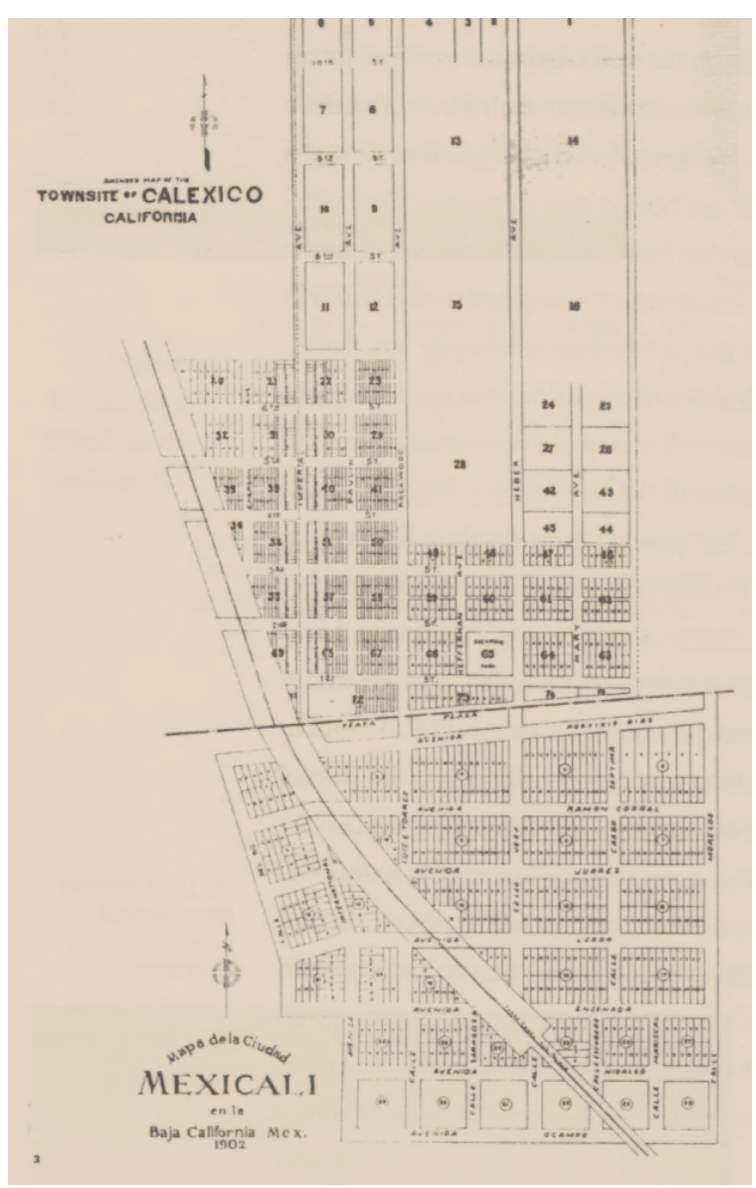

Figura 12. En el plano de 1902 incluye las dos poblaciones, se aprecia la frontera en línea horizontal y en línea vertical poco curveada la línea de ferrocarril. Las trazas coinciden con algunas calles, la lotificación cuadriculada y más dividida en Estados Unidos y rectangular y un poco menos dividida en México. Fuente: Libro "Ciudades mexicanas del siglo XX" de Héctor Quiroz Rothe, editado por UNAM.

"La notoria ausencia de la plaza central, relevante en las urbes mexicanas y que funciona como núcleo físico y social de la ciudad novohispana. Una característica del proyecto de Mexicali es la coincidencia de algunas calles con otras de la vecina Calexico en Estados Unidos, quedando aún sin resolver la forma como se incluyó la vía del ferrocarril que atraviesa diagonalmente la traza, segmentando con ello buena parte de las manzanas $e$ induciendo a su irregularidad; de esta manera, se plantea la posibilidad de que la vía del Intercalifornia fuera sobrepuesta arbitrariamente sobre un plano original, lo cual se sustenta en el hecho de que el ferrocarril llego a Mexicali en 1904, un año después de que Rockwood realizara el proyecto urbano" (Corona, 1998).

Los trazados urbanos que surgen a través de la implantación del ferrocarril dan una configuración diferente a la organización del espacio (Figura 12) ya que alrededor siempre se genera una actividad económica importante. En el caso de Mexicali la agroindustria que se desarrolló en la región fue lo que lleva a convertirla en la ciudad exportadora de algodón más importante del mundo y una sofisticada comunidad agrícola probablemente controlada y administrada desde Calexico en el lado norteamericano.

El trazo original de Mexicali (Figura 13) estaba conformado por 460 lotes de dimensiones variables contenidos en 32 manzanas, delimitadas entre siete avenidas dispuestas oriente-poniente, y por seis calles principales y tres calles secundarias. En el extremo norte del polígono el trazo iniciaba en la línea internacional, al sur terminaba en la avenida Ocampo, al oriente en la calle Morelos y 
hacia el poniente en la calle del Rio; las calles y avenidas tenían un ancho de 30 metros, predominaban los terrenos de 15 metros de frente por 60 metros de profundidad (Robles, 2006).

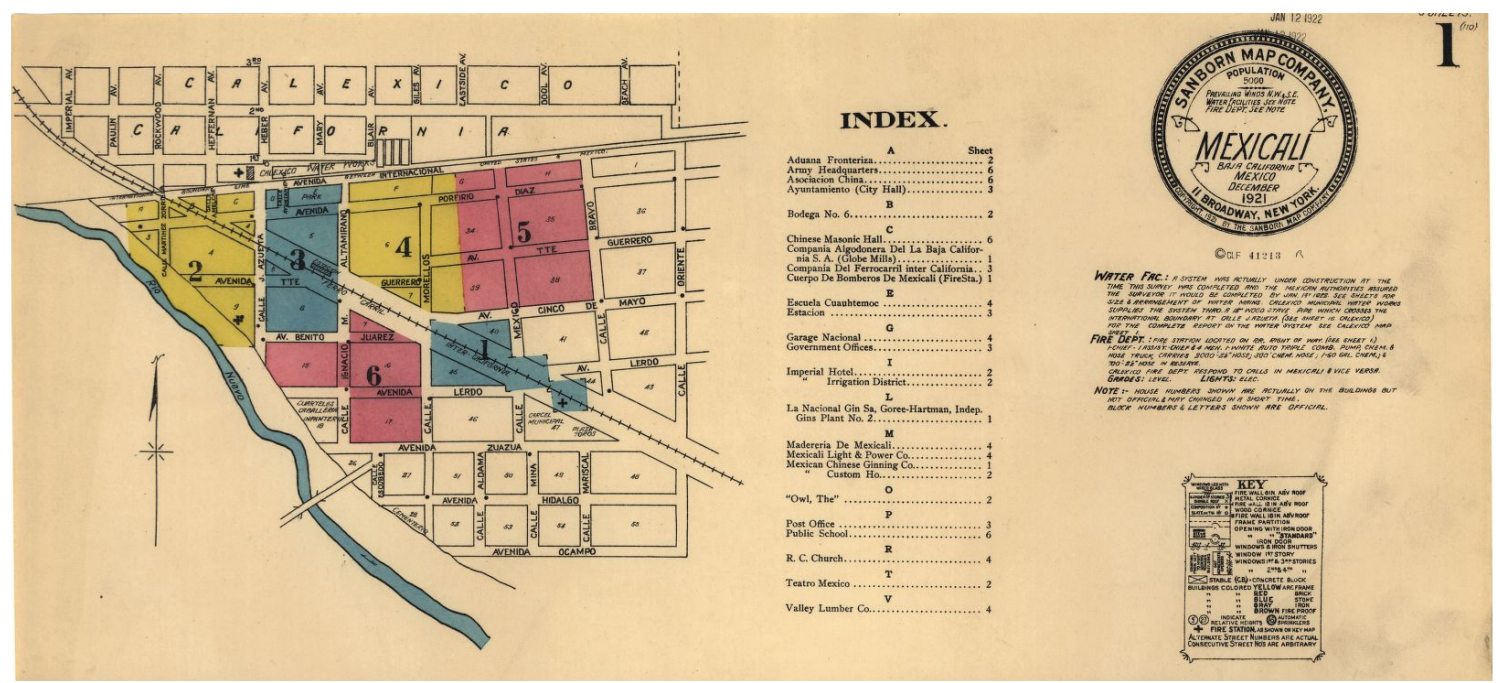

Figura 13. Plano de Mexicali y parte de Calexico de 1921. Fuente: Biblioteca del Congreso de Estados Unidos.

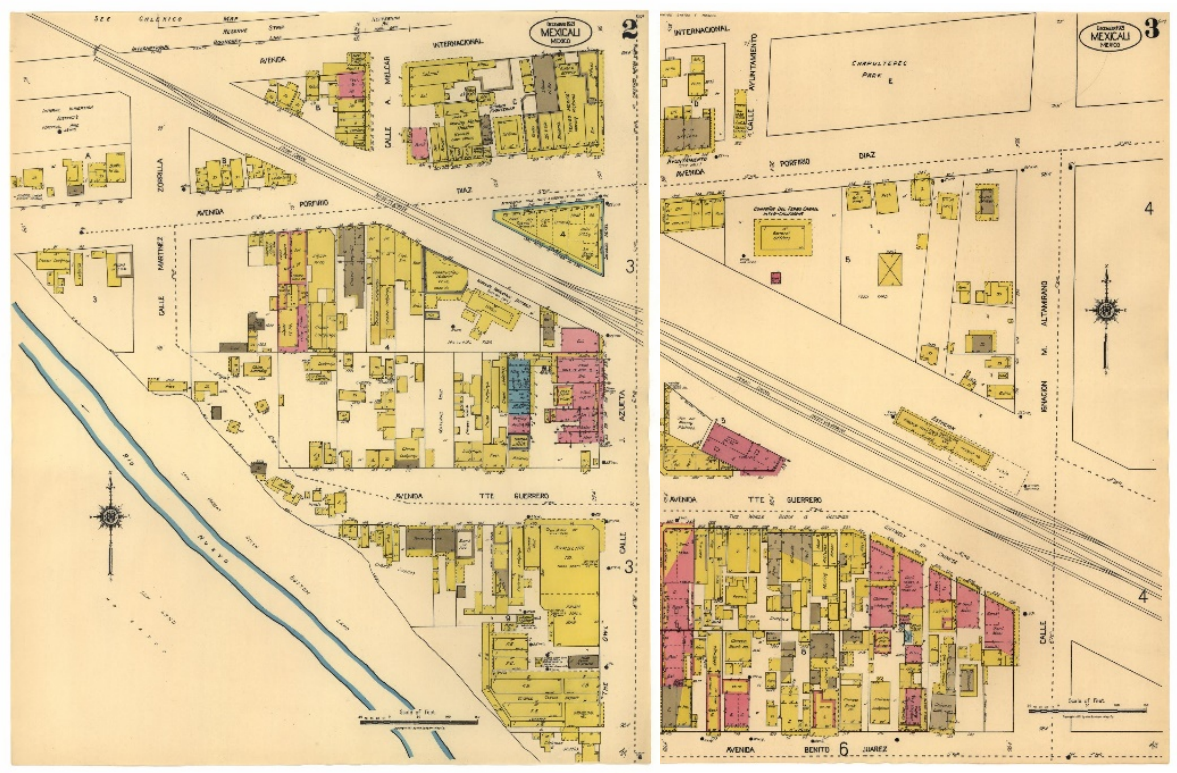

Figura 14. Plano de Mexicali y su lotificación de 1921 (Barrio chino). Fuente: Biblioteca del Congreso de Estados Unidos.

De ese primer trazo urbano aún subsisten seis calles: Lerdo, Juárez, Hidalgo, México, Melchor Ocampo y Morelos. Conforme la ciudad se fue expandiendo, las principales actividades comerciales se mantuvieron en ese primer cuadro que quedaba delimitado hacia el norte por la frontera y al oeste por un barranco artificial, excavado a fuerza de dinamita en el intento de controlar la inundación de 1905.

"El derecho de vía del ferrocarril destaca como eje vial predominante en el poblado, con una superficie que en 1907 ocupaba el 20\% del área total del trazo urbano, en torno a este espacio, con el posterior auge algodonero, se empezó a desarrollar la primera arquitectura industrial de la ciudad. Con la llegada del ferrocarril, Mexicali mostro un cambio importante en su arquitectura debido a la importación de edificios prefabricados de madera (sistema constructivo balloon frame). Esta situación vino a darle una apariencia más parecida a los pueblos del oeste de Estados Unidos ${ }^{2}$ que al de una ciudad

\footnotetext{
2 Todos presentan una preferencia a la estructura cuadriculada de sus trazados, estas cuadriculas urbanas formaban parte de una estrategia colonizadora más general, ya que los Estados Unidos comenzaron disponiendo la retícula a lo largo de todo el territorio (delimitando municipios, condados o estados), de forma que las ciudades eran la concreción construida en determinados puntos seleccionados.
} 
mexicana. La imagen urbana original predominante conformada por pocos edificios de adobe, se modificó con la presencia de inmuebles de madera, lo cual habla del fenómeno multicultural inherente a la localización fronteriza de Mexicali, en donde el impulso de fundación y crecimiento inicial no podrían explicarse sin la presencia de capital extranjero" (Robles, 2006).

Un rápido recuento de la historia del centro de Mexicali es la de construcción y reconstrucción. Se quitan edificios industriales y se construyen otros o ningunos, algo lamentable sin vuelta atrás para su patrimonio. Durante la década de 1920 la población se vio favorecida por la Ley Volstead o Ley $\mathrm{Seca}^{3}$, lo que provoca la proliferación de construcciones como centros nocturnos, casinos, cantinas y cabarets a lo largo de la frontera para cubrir la necesidad de esparcimiento de los norteamericanos.

En el centro se empezaron a construir en esa época cafés, restaurantes, casas de cambio y tiendas, mezclados con bodegas de mercancías, bancos y oficinas del ayuntamiento. Algo significativo de este primer trazo es que debajo de los edificios se construyeron más de 100 túneles subterráneos que conectaban hacia el otro lado de la frontera donde la mafia china controlaba de forma clandestina casinos y todo tipo de negocios ilícitos.

En 1923 un incendio dejó en descubierto las actividades subterráneas de los chinos en Mexicali y el lugar se abandonó. La venta de predios a mexicanos y la clausura de los pasadizos paulatinamente dejaron a estas manzanas y a los edificios en ruinas y sin uso. En 1960 con un segundo incendio no quedó mucho y el centro se fue abandonando y degradando poco a poco.

A mediados del siglo XX cuando la ciudad ya se había expandido a lo largo de la frontera con su retícula regular, el periodista Fernando Jordán describe su llegada a Mexicali:

"Es una ciudad moderna y limpia, si descartamos esa fatalidad irremediable del polvo. Su trazo es amplio y seguro, y a pesar de haber crecido de la noche a la mañana, en la improvisación no perdió cualidades urbanísticas. Sus calles son anchas y sus edificios, aunque modestos, son discretos en cuanto a gusto. No podría decirse que el centro de la ciudad sea bello: tiene demasiado movimiento, demasiados comercios de toda clase. Los chinos. Que merecen una mención en la construcción de la ciudad y en la colonización del valle, han impreso un sello internacional en la zona urbana central, donde cada metro de terreno está destinado a una función necesaria para las transacciones comerciales" (Jordán, s./d.).

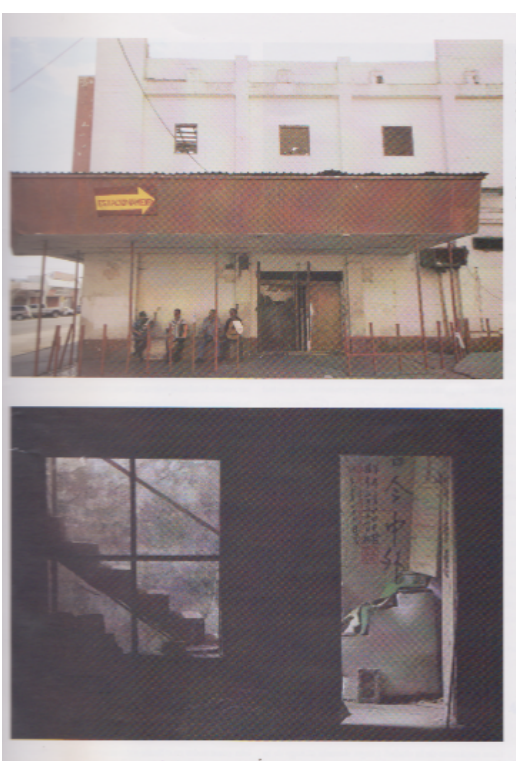

Así, el centro de la ciudad sufrió sus primeras crisis entre 1974 a 1994 con la mudanza de los servicios gubernamentales y los grupos empresariales más importantes a mejores lugares en sectores modernos de la ciudad. Luego, durante el auge de la industrialización, la llegada de las maquiladoras cambió de forma definitiva los focos de actividad económica (Correa, 2014). Actualmente, el centro parece un lugar que no termina de verse abandonado, contiene edificios vacíos y manzanas demolidas que ven pasar el tiempo en una de las fronteras más activas del mundo.

Figura 15. El artículo de la revista Tierra Adentro "Cómo se perdió el centro de Mexicali, de la ciudad subterránea al abandono. De Elma Correa y fotografías de Paulina Sánchez. Fue de gran ayuda para tener una idea antes de visitar el sitio. Arriba la primera tienda de abarrotes de la ciudad hoy en abandono. Abajo un sótano utilizado como casino clandestino.

\footnotetext{
${ }^{3}$ La Ley Volstead o Ley Seca promulgaba la prohibición de la venta, producción y consumo de bebidas alcohólicas en territorio estadounidense.
} 


\section{Consideraciones Finales}

La ciudad de Mexicali es un claro ejemplo de como un pequeño trazado diseñado por una compañía se convierte, en sus inicios, en el rancho algodonero más grande del mundo. Hoy en día, es una de las ciudades de más rápido crecimiento en el norte de México, quizás, el modelo de company town más exitoso del país. Ayudando a consolidar urbanísticamente la frontera, caso contrario a lo que sucede en el lado norteamericano con la ciudad de Calexico ya que no creció tanto como Mexicali.

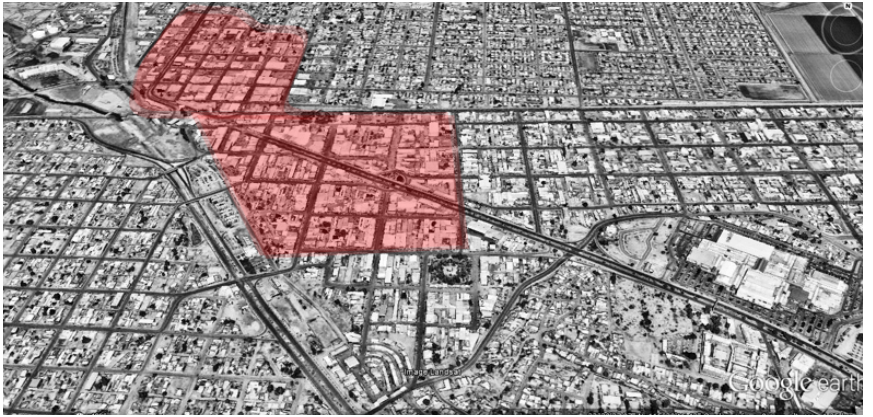

Figura 16. Fotografía aérea señalando las áreas de oportunidad del primer cuadro de las dos poblaciones entre los dos países.
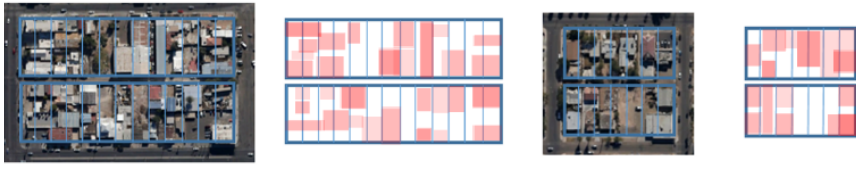

Figura 18. En el trazado de Calexico en el lado estadounidense se aprecia una manzana tipo de $100 \mathrm{~m} \times 115 \mathrm{~m}$ aproximadamente, incluyendo su callejón en el lado más largo, unas manzanas más cuadradas que las rectangulares en el caso de Mexicali. En el lado mexicano, las manzanas eran aproximadamente de $180 \mathrm{~m}$ x 130m, también con su callejón en su lado más largo. Cabe mencionar que algunas de las calles coinciden a pesar de la frontera entre los dos países.

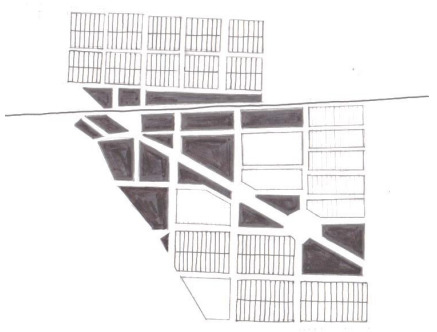

Figura 17. Esquemas de ocupación de las manzanas en los dos lados de la frontera. Las avenidas tienen un ancho de $30 \mathrm{~m}$ y la lotificación predominan terrenos de $15 \mathrm{~m}$ de frente por $60 \mathrm{~m}$ de profundidad.

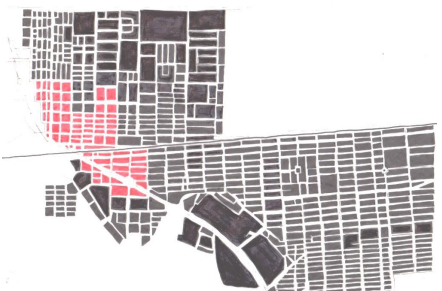

Figura 19. Esquemas y dibujos de la traza urbana de Mexicali y Calexico, en rojo se señala los centros más antiguos, localizados en base a los planos fundacionales de las compañías a principios del siglo XX.

Esta investigación pone en evidencia que muchas de las construcciones industriales que se creía que aun existían fueron demolidas. Se confirma que ya no existe nada del área industrial inicial en el centro de la ciudad. Hace falta una propuesta para proteger lo poco que queda del patrimonio industrial reconociendo los trazados más antiguos, buscando proyectos integrales donde los edificios abandonados puedan ser utilizados como motor de reactivación económica. El economista español experto en patrimonio industrial Miguel Ángel Álvarez Areces (2011) menciona:

Actualmente el patrimonio industrial es considerado un nuevo bien cultural. Las regiones y las ciudades donde la crisis industrial afectó profundamente son el punto de inflexión donde se comienza a trazar y valorar este tema. Se trata en muchos casos de convertir la necesidad en virtud, cobrando interés creciente en políticos locales, movimientos ciudadanos, defensores del patrimonio y de la arqueología industrial, proponiendo una nueva mirada de arquitectos e ingenieros que ponen su objetivo en el territorio urbano. (Álvarez Areces, 2011).

Así pues, los gobiernos locales y las políticas actuales de preservación deberán encarar el siglo XXI generando nuevos proyectos de regeneración de trazados e instalaciones industriales para, de esta forma, garantizar los valores y la identidad urbana, en este caso en particular, de la península de Baja California.

El antecedente más próximo de regeneración es el emprendido en el centro histórico, en donde se peatonalizaron calles y se crearon recorridos culturales y turísticos — promovidos por Rubén 
Hernández Chen ${ }^{4}$ - por los túneles subterráneos del barrio La Chinesca. Por un lado, se ha invertido en mejorar la imagen del centro, pero por otro, no se ha llegado a una consolidación por el estado grave de abandono y deterioro de las construcciones existentes.

Es necesario ampliar y difundir investigaciones sobre la historia reciente del urbanismo industrial en México. Se percibe cierto desconocimiento sobre el surgimiento de este tipo de ciudades, las cuales, poseen una identidad reciente. A veces, por su pronta modernidad no son tomadas en cuenta, pero no por ello son menos valiosas en términos de apropiación del territorio, incluso cuando éstas han sido diseñadas por compañías extranjeras. Es importante estudiar y mostrar los proyectos que hicieron de México un país moderno y ayudaron a consolidar la frontera norte, haciendo énfasis en la manera en que se desarrolló la región.

Por tanto, es necesario crear propuestas de revitalización de edificios de la era industrial. Desde esta consideración se presentan tres oportunidades a futuro:

1. Organizar y reconocer el alcance del territorio para mostrar la arquitectura de ese periodo histórico, buscando la recuperación de la identidad a través de resaltar lo que está ahí en ruinas.

2. Determinar su estado de conservación, verificar la situación de la tenencia de los terrenos abandonados (buscar normativas de como el municipio puede manejar las propiedades intestadas para uso de proyectos de regeneración urbana), mejorándolo tanto en su imagen como en su interacción con la población del centro y el turismo.

3. Valorar la importancia del rico legado que es el centro de Mexicali para buscar una reactivación real, económica y visualmente mejorada a través de nuevas y distintas actividades para beneficio de la comunidad, haciendo que la sociedad se involucre en el mejoramiento paulatino de los espacios. La participación ciudadana ayudaría a potenciar el centro como ya se ha visto recientemente, integrando sus actividades y vinculándolas al nuevo desarrollo.

Se percibe que la arquitectura industrial del centro de Mexicali pretendiera ser borrada, ya sea por las fuerzas de la naturaleza, como en la inundación de principios del siglo XX y los terremotos a principios del siglo XXI, o por la mano del hombre como se ha visto en los últimos años.

La sensación que se tiene al recorrer sus calles en su trazo más antiguo es de un lugar abandonado y descuidado. Varias entrevistas con ciudadanos jóvenes confirmaron que gran parte de la población no se identifica nada con esta parte de la ciudad y es aquí donde toma importancia la identidad urbana de la población, que en estos momentos está perdida.

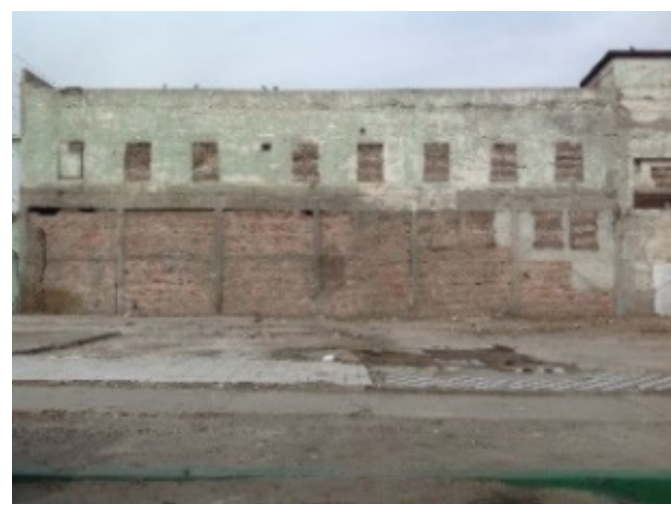

Figura 20. Fotografía del autor que muestran la decadencia del centro de Mexicali.

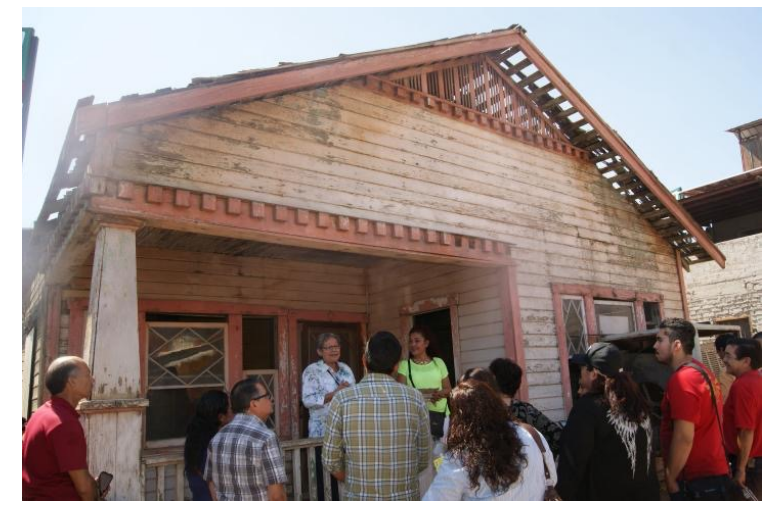

Figura 21. El título de la noticia: Emerge antigua vivienda en medio de la modernidad. Aparece casa rústica de antes de 1925 de madera en centro histórico luego de la demolición de algunos edificios. Fuente: Periódico el Mexicano, artículo por Eduardo Villa, 2014.

\footnotetext{
${ }^{4}$ Rubén Hernández Chen es dueño de una tienda local y director del Comité del Centro Histórico de Mexicali. Chen es reconocido por sus esfuerzos por promover las tradiciones chinas en Mexicali, que incluyen estos recorridos por La Chinesca.
} 
Habría que decir también que la aportación personal de este estudio es hacer una llamada de atención a la situación de olvido que sufre el centro de Mexicali, ya que la capital del Estado de Baja California no puede seguir con esta imagen de degradación arquitectónica en su centro urbano.

La reflexión final que nos queda es seguir con la búsqueda de los vestigios que hicieron surgir a ésta ciudad que no se entiende sin su vecina norteamericana y sugerir un plan de reconocimiento del patrimonio industrial entre Mexicali y Calexico, recopilando información de los edificios de las compañías, los tanques elevados, las viviendas, almacenes y las bodegas que aún quedan de ese inicio industrial de las poblaciones.

Como dato sorprendente, en el año 2014 al demoler una construcción del centro de Mexicali se descubrió una casa que databa del año 1925, fabricada con el sistema constructivo balloon frame; por ahí, es por donde se podría empezar a narrar la historia industrial, porque Mexicali nació industrial y aunque su centro moderno no lo parezca, es patrimonial.

\section{Referencias}

Acosta, A. (2014). El modelo Company town y Pedro Cornu: discurso de desarrollo fabril y espacial en Mexico (1861-1913). Labor \& Engenho, 8 (2), 101-111, abr./jun., Campinas [SP] Brasil.

Aguirre Bernal, C. (1983). Compendio histórico biográfico de Mexicali 1539-1966 (vol.1). Mexicali [México]: sin editorial.

Álvarez Areces, M. Á. (2011). Conservación y restauración del patrimonio industrial en el ámbito internacional. Abaco, Revista de cultura y ciencias sociales. 2da época No. 70, pp. 22-39. Gijón [Asturias] España.

Anguiano, M. E. (1992). Irrigación y capital para transformar el desierto. Formación social del valle de Mexicali principios del siglo XX. Frontera Norte, 4 (8), jul./dic. 1992.

Bernal Rodríguez, F. A. (2002). Mexicali: 100 años de agua y vida (pp.17-32). En Héctor Manuel Lucero Velasco (coord.) Mexicali, 100 Años: arquitectura y urbanismo en el desierto del Colorado. México DF: Grupo Patria Cultural.

Corona, E., Arredondo, J., \& Rojas y Martinez, F. (2013). Mexicali una ciudad sin valoración histórica de su patrimonio cultural (p. 814-838). International Conference Virtual City and Territory. $9^{\circ}$ Congresso Città e Territorio Virtuale. Roma [Italia]: Università degli Studi Roma.

Correa, E. (2014). Como se perdió el centro de Mexicali, de la ciudad subterránea al abandono. Revista Tierra Adentro, México DF, Consejo Nacional para la Cultura y las Artes, septiembre 2014.

Griffin, J. (2012). Las inundaciones, las batallas con el Rio Colorado para salvar los valles Imperial y Mexicali. Revista El Rio (revista de historia regional de Mexicali y su valle), Año V, n.16, abr./jun., Mexicali [México].

Grijalva, Aidé. (2014). “Agroindustria y algodón en el valle de Mexicali. La compañía industrial jabonera del pacifico". SCIELO, Estudios fronterizos, 15 (30), jul/dic., Mexicali [México].

Hendricks, W. (1996). Guillermo Andrade y el desarrollo del delta mexicano del rio colorado 1874-1905 [Colección Baja California : Nuestra Historia]. Mexicali [México]: Universidad Autónoma de Baja California.

Jordán Juárez, F. (2010). El otro México. Biografía de Baja California [Colección Baja California: Nuestra Historia]. Mexicali [México]: Universidad Autónoma de Baja California. (obra original publicada em 1956).

Kering, D. (2001). El valle de Mexicali y la colorado River Land Company 1902-1946 [Colección Baja California: Nuestra Historia]. Mexicali [México]: Universidad Autónoma de Baja California.

Ley, J., \& Fimbres, N. (2011). La expansión de la ciudad de Mexicali: una aproximación desde la visión de sus habitantes. Región y Sociedad, 23 (52). set./dic., Hermosillo [México].

(C) Labor \& Engenho, Campinas [SP] Brasil, v.10, n.4, p.343-356, out./dez. 2016. 
Ley, J., \& Calderon, G. (2008). De la vulnerabilidad a la producción del riesgo en tres primeras décadas de la ciudad de Mexicali, 1903-1933. Región y Sociedad, 20 (41), ene./abr., Hermosillo [México].

Ley, J., Ortega, L., Fimbres, N., \& Ortega, G. de los Á. (2012). .Mitos en el valle de Mexicali: una cartografía de lo intangible. Revista de Geografía Norte Grande, 52, set., Santiago [Chile].

Méndez, E. (2000). Ciudades y arquitecturas del noroeste mexicano". Revista Región y Sociedad, 12 (20). El Colegio de Sonora. Hermosillo [México].

Morales, H. (2014). Editorial L\&E, v.8, n.4, 2014 : Dossier Company Towns. Labor \& Engenho, 8 (4), 3-4, out./ dez., Campinas [SP] Brasil.

Palacios, F. (2015). La colonia Lerdo y su núcleo poblacional: Ciudad Lerdo. Divulgación Dhiré, Divulgación de las historias regionales de Baja California.

Padilla, A. (1998). Inicios Urbanos del Norte de Baja California, Influencias e ideas, 1821-1906. Mexicali [Baja California] México: Universidad Autónoma de Baja California.

Peimbert Duarte, A. J. (2010). Recuperar las ruinas: espacio público y prospectiva del paisaje post-industrial de Mexicali. International Conference Virtual City and Territory. 6to. Congreso Internacional Ciudad y Territorio Virtual. Mexicali [México]: Universidad Autónoma de Baja California.

Piñera, D., \& Bejarano, A. (2011). Expresiones arquitectónicas compartidas en la frontera de Baja California y California. Revista Culturales, 7 (14), Mexicali [Baja California] México.

Piñera, D. (2006). La Frontera Norte: de la Independencia hasta nuestros días. En Á. Matute, \& C. Vázquez Mantecón (Eds.), Estudios de Historia Moderna y Contemporánea de México (v.12, pp.27-50). México DF: Universidad Nacional Autónoma de México, Instituto de Investigaciones Históricas.

Pozo, B., Calderon, B., \& Pascual, H. (2009). Recuperar y rehabilitar el patrimonio industrial urbano. Entre el desamparo institucional y la voracidad urbanística. Ciudades, 12, 197-219. Valladolid [España].

Quiroz, H. (2008). Ciudades Mexicanas del siglo XX. México DF: Universidad Nacional Autónoma de México.

Robles, C. (2006). La arquitectura de Mexicali, Orígenes. Mexicali [México]: Universidad Autónoma de Baja California.

Sabaté, J. (2004). Paisajes Culturales. El patrimonio como recurso básico para un nuevo modelo de desarrollo. Urban 9. Primera época, pp. 8-29. Barcelona [Cataluña] España.

Starr, K. (1991). Material Dreams: Southern California through the 1920s. New York: Oxford University Press. 